\title{
INTEGRATED SIMULATOR OF MOBILE AD-HOC NETWORK-BASED INFRASTRUCTURE : A CASE STUDY
}

\author{
Aznam Yacoub \\ Polytechnique Montreal, Heterogeneous Embedded System Laboratory, Montreal, QC, Canada \\ aznam.yacoub@polymtl.ca
}

\begin{abstract}
Mobile Adhoc Network-based (MANET-based) infrastructures have been proposed as communication support in case of disastrous events and emergency situations. Indeed, standard communication systems like cellular networks might become unavailable or entirely destroyed. However, designing a reliable network which various heterogeneous systems will operate on is a well-known and costly problem. Simulation represents a good alternative for checking properties on these networks, but simulators usually lack confidence or accuracy. Therefore, an integrated simulator architecture of MANET-based network has been proposed. This paper illustrates the implementation of this kind of architecture, and discuss the pros and cons of this kind of solution.
\end{abstract}

Keywords: DEVS, Mobile Ad Hoc Networks, Verification and Validation, Disaster Response.

\section{INTRODUCTION}

Mobile Ad Hoc Networks (MANETs) have been proposed in the litterature (Kiess and Mauve 2007, Reina et al. 2015, Mohammed and Al-Ghrairi 2019) as a communication technology in the case of emergency and disasters. Indeed, cellular-based infrastructures might become unoperational, for instance in the case of earthquake like in Haiti. More important, rescue teams (police, firemen, etc.) and citizens might use several different isolated networks and protocols or devices (Android, iPhone, etc.), making the communication and synergy between people harder. While MANETs can be quickly deployed without fixed infrastructure, setup or prior requirements, their flexibility is attractive when communications between victims and rescue teams are crucial. However, their implementations face an important challenge: their accuracy and their reliability. Indeed, this kind of networks has to been proved before being deployed, but their verification cannot be done in real situations.

Therefore, simulators appear as an inexpensive manner to evaluate the performance and the accuracy of algorithms and systems in virtual and theoritical scenarios (Manpreet and Malhotra 2014). However, despite the increasing numbers of simulators, the credibility of their results decreased over the time (Kurkowski et al. 2005, Hogie et al. 2006). Among the problems encountered during the development of MANETs, some are inherent to simulation in general: simulations are developed for a specific purpose and not for checking all the properties of all the subsystems using a unique model (Sargent 2001). Particularly, MANET simulators generally focus only on the protocol reliability, but the used scenarios for their validation imply simple applications (Andel and Yasinsac 2006).

Yacoub (2019) states that MANET-based ecosystems may be widely heterogeneous, with different kinds of systems (real-time, non real-time, autonomous, controlled, etc.). Their nature implied also hetereogeneity 
of their models during design, verification and validation $(\mathrm{V} \& \mathrm{~V})$ processes. Building a general simulator which integrates all these models with their own granularity for increasing their reliability is therefore a challenge. That's why we proposed (Yacoub 2019) some hints and insights for building a simulator for MANET-based infrastructure which is able to integrate simulators with different paradigms and granularity using the Discrete-Event System Specifications (DEVS) theory (Zeigler, Muzy, and Kofman 2019), and especially the DEVS-Bus concept (Kim and Kim 1998).

In this paper, we illustrate this approach through the building of such a simulator, which integrates NS3 (Riley and Henderson 2010) and OMNET++ (Varga and Hornig 2008). The first section makes some recalls about our proposed architecture. The second section introduces the implementation. The last section presents some results of simulation using our architecture, and discusses about the advantages and drawbacks of this approach.

\section{RELATED WORKS}

Our work mainly relies on the Virtual Communication Stack (VCS) architecture (Yacoub 2019), and the Theory of Modelling and Simulation (TMS) (Zeigler, Muzy, and Kofman 2019). The first part of this section recalls some important points about the foundation of our proposed architecture, while the second part recalls its characteristics.

\subsection{DEVS Methodology for Modelling, Simulation, Verification and Validation}

TMS (Zeigler et al. 2019) gives guidelines for formalizing, modelling and simulating systems in a hierarchical, uniform and universal way. Especially, the VCS intensively uses the DEVS formalism which separates the conceptual model described using an algebraic structure, and the simulation model represented by the DEVS Abstract Simulator.

DEVS Atomic Model. At the component level, a Classic DEVS atomic model is a tuple

where

$$
A=<X, S, Y, \delta_{i}, \delta_{e}, \lambda, t a>\text {, }
$$

- $\quad X$ and $Y$ are respectively the (possibly infinite) set of input and output events;

- $S$ is the set of internal states;

- $\delta_{i}: S \rightarrow S$ is the internal transition total function;

- $\delta_{e}: Q \times X \rightarrow S$ with $Q=\{(s, e), s \in S, e \in[0 ; \operatorname{ta}(s)]\}$ is the external transition total function. $Q$ is called the total set and $\delta_{e}$;

- $\lambda: S \rightarrow Y$ is the partial output function;

- $t a: S \rightarrow \mathbb{R}^{+}$is the lifespan function.

Intuitively, a DEVS atomic model represents a system which remains stables between two occurences of event, and formalized by a state-machine which stays in a current state $s$ during at most $t a(s)$ units of time. Events can come from the environment (reactive behaviour), or can be generated by the system itself (autonomous behaviour). In any cases, the event is well-dated. After the end of the lifespan, the transition $s^{\prime}=\delta_{i}(s)$ is enabled. An ouput $\lambda(s)$ is emitted. When the event is fully computed, the model changes its state to $s^{\prime}$. If an event $x$ is received at time $e$ before the end of the lifespan $t a(s)$, the model changes its state to $s^{\prime}=\delta_{e}(s, e, x)$. We call transitory state any state $s$ for which $t a(s)=0$, and passive state any state $s$ for which $\operatorname{ta}(s)=\infty$.

DEVS Coupled Model. At the compound level, a Classic DEVS coupled model is a tuple

$$
M=<X, Y, D,\left\{M_{d} \mid d \in D\right\}, E I C, E O C, I C, \text { Select }>\text {, }
$$

where

- $X$ is the set of input ports; a port is defined by a set $X_{p}$ of events it accepts; 
- $Y$ is the set of output ports; a port is defined by a set $Y_{p}$ of events it emits;

- $D$ is the set of components; $d \in D$ if $d$ is a DEVS atomic model, or a DEVS coupled model;

- $M_{d}$ is the set of instances of components; $D$ represents the structures, whereas $M_{d}$ represents each instance of these structures;

- $\quad E I C, E O C, I C$ are respectively the external input coupling, the external output coupling and internal coupling relations;

- Select is the tie-breaking function.

Intuitively, input events are dispatched from the coupled structure to the internal components following the EIC relation. Changes are computed inside each compound, internal events are triggered and dispatched using the $I C$ relation. Finally, output event are dispatched outside the compounds following the EOC relation. The Select function allows choice of the processed component in the case of simultaneous events. Indeed, Classic DEVS prevents events happening in the same time, and enforces deterministic behaviours.

DEVS Simulation Models and Algorithms. Abstract simulation models define the behaviour of the DEVS structure. A DEVS simulation model is a tree of simulators and coordinators which follows the compositionl structure of the conceptual model, at the right granularity level. To each DEVS atomic model corresponds a DEVS simulator which is working as follow:

- When initialization message is received, it computes its current state according to the conceptual model and the date of the next internal event using the $t a$ function;

- When an internal transition message is received, it emits an output event to its parent coordinator, proceeds the internal transition and computes the date of the next internal event using the $t a$ function;

- When an external transition message is received, it proceeds according to the external transition table, and computes the date of the next internal event using the $t a$ function.

To each DEVS coupled model corresponds a DEVS coordinator which is working as follow:

- Initialization message is dispatched by the coordinator to its children. All the dates of next events are retrieved and sorted by ascending order.

- When an internal transition message is processed, it picks the first event in the event queue and emits it to corresponding children; dates are updated and the event queue sorted again;

- External transition message from another compound are dispatched to the receivers according to the EIC function. The event queue is then recomputed after the updating of all the internal components;

- Output received from one of its component is dispatched to the parent coordinator according to the EOC function; Corresponding external transition message is emitted to the internal receivers using the $I C$ function.

Finally, the DEVS formalism is itself composed by a hierarchy of formalisms (Giambiasi 2009) which allows modellers to increase or decrease the level of details.

\subsection{DEVS-Bus: How to Couple non-DEVS Model with DEVS Model}

Heteregeneous simulation refers to combination of simulators developed in different languages and environments, or paradigms, and which work in an interoperable way to achieve a general simulation. The main adressed problem is that such interoperation needs data exchange and time synchronization between the simulators. Kim and Kim (1998) developed a DEVS-Bus, an interface which provides an unified simulation protocol based on DEVS, in order to resolve these two questions. Each simulator is associated to a protocol converter which transforms this simulator into a DEVS-compliant simulator.

Definition 1. A DEVS-compliant simulator is a model whose the conceptual model can be defined using a DEVS algebraic formalism, and whose the computerized simulation follows the DEVS abstract simulator algorithm. 
Therefore, a non-DEVS Simulator encapsulated with a DEVS-Bus becomes a DEVS-compliant model. Knowing if the resulting simulator is equivalent to the prior one is out of the scope of this paper. However, the DEVS-Bus theory gives an important result for our architecture of simulation of MANET-based infrastructure: any MANET simulator can be used in a more wide simulation environment and is able to communicate with other simulators without reimplementing all the models.

Moreover, advanced and well-known simulators like NS3 and OMNET++ use a Discrete-Event Architecture, which makes the interoperability easier. In other words, we can build a simulator based on existing network simulators and which will make checking of end-user application and devices possible.

\subsection{Virtual Communication Stack}

Our architecture relies on a virtual communication full-stack (instead of a classical three-layer model in MANET simulator) which adresses problems encountered by network simulation, and fullfills following goals:

- Achieve MANET-based simulation by using existing models instead of recreating specific model per implementation.

- Take into account the specificities of each component of the infrastructure by achieving heterogeneous simulation;

- Improve the accuracy of the simulation by executing real software during the simulation and final scenarios, and validating both the simulation model and the infrastructure using test cases and use cases;

- Swap between Simulation, Emulation, and Execution without redesigning and redevelopping software thanks to a common interface;

- Allow the choice of the good level of abstraction while developping the simulator;

- Bring strong basis for V\&V of MANET simulation models and for V\&V of the resulted infrastructure.

The VCS acts as a proxy between the end-user application and the operating system (Figure 1). It handles connections between devices, routing protocols and communication with external networks as a VPN middleware would do. It is embedded in one or several services installed on each device, and which enable the access to the MANET. These services can be distributed on different devices. Devices can be embedded systems, drones, computers, smartphones, etc. working on different operating systems (Windows, Android, Linux, iOS, MacOS, etc.). Implementations of the VCS may differ according to the platform: a TUN/TAP driver, a software service, etc. From a high-level point of view, the behaviour of the stack is formally defined. Communications between software through the network is done thanks to virtual sockets which connects devices as usual. Data transmission is totally transparent for the end-user application.

For the remaining sections, we provide some definitions.

Definition 2. A device is any piece of hardware in which the VCS is embedded or any piece of hardware which connects to the network. A VCS service is a part of the VCS. A VCS service handles one or several layers of the stack. A VCS software is any piece of software which communicates through the network. A Client/Server is any couple of software which works under the producer/consumer design in the usual definition.

Once the VCS runs on a device, communication is done through the OS stack and through the virtual stack. Message can go through all the layers or is directly routed to the corresponding layer of the OS stack. While the VCS is designed using an event-based architecture, it is easily encapsulated in a DEVS-compliant model.

Especially, we define our DEVS structure with: 


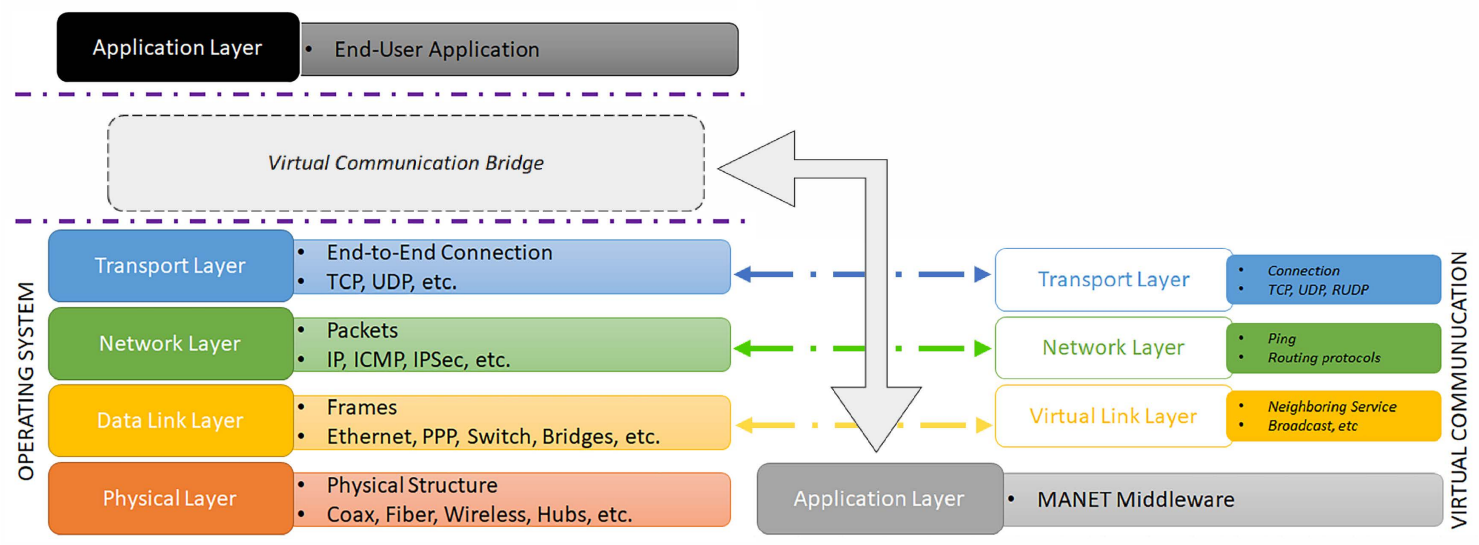

Figure 1: The Virtual Communication Stack. Traffic can be routed through the entire stack or directly routed to the corresponding layer of the OS Stack.

- $X$, the set of input events is defined by the messages carried by the communication protocol between the application and the stack on the one hand, and the stack and the OS on the other hand;

- $Y$, the set of output events is as the set of input events;

- $S$, the set of states is defined by the states of the VCS after executing each instruction;

- $\delta_{i}$ is the transition function when an instruction shall be executed by the stack (including neighbors discovering);

- $\delta_{e}$ is the transition function when the stack shall perform operations related to an external message (including multithread synchronization);

- $\quad t a$ is the time advance function which corresponds to the time between the execution of two instructions or possibly the time during a specific thread is sleeping (if the middleware is multithreaded).

The VCS proxy then defines at least events for: sending message, receiving message and synchronization mechanisms (thread sleeping, thread awakening, shared memory access, etc.). We integrate this stack in a DEVS-compliant environment for verification and validation of MANET-Based Infrastructure using NS3 and OMNET++.

\section{INTEGRATED ARCHITECTURE FOR VERIFICATION AND VALIDATION OF MANET- BASED INFRASTRUCTURE}

We recall the purpose of our architecture is to take advantage of the MANET simulator for checking properties about protocols and mobility, using the real end-user applications which are used in real conditions of emergency. In order to achieve this environment, we use NS3 and OMNET++ for the simulation of the physical part, and the VCS for the logical part and the routing. Finally, this will allow us to verify and validate the reliability of our devices and software when they communicate through a specific MANET, but also the reliability of the MANET middleware. We recall also that this approach can be developed with any kind of simulators, if they are encapsulated in a DEVS-Bus in order to make a DEVS-compliant model.

\subsection{VCS Simulation Environment}

The first part is the VCS Simulation Environment (Figure 2a) whose the role is to initialize the DEVS simulation environment and to handle the main clock of the simulation. This environment instantiates one or several instances of the real implementation of the VCS, while the underlying Bridge translates the messages from the OS or from the VCS into understandable events for the other simulators using a protocol converter. The DEVS-Bus Network Stack is responsible of the synchronization of the VCS Middleware if needed. Indeed, since the VCS Middleware is executed, the time of execution can differ from the simulated 
time of the remaining architecture. If the simulator is run in emulation mode or execution mode, the DEVSBus Network Stack dispatches immediately each message (in this configuration, it is only composed by transitory states).

\subsection{OMNET++ and NS3 Integration}

The second part of our architecture concerns the integration of existing MANET simulators in order to simulate the physical layer or the OS Stack inside the OS DEVS Model. In this example, we choose NS3 (Riley and Henderson 2010) and OMNET++ (Varga and Hornig 2008) because they both implement a discrete-event approach close to the DEVS formalism. Indeed, these both simulators implement hierarchies of models, Message concepts which are a mix between events (in simulation) and data (in communication), Channel concepts which are a mix between concept of ports in simulation and sockets in communication, and a discrete-event scheduler. Moreover, one the one hand, OMNET++ is a well-used simulator (Mallapur and Patil 2012, Manpreet and Malhotra 2014) developed since 1997, which supports parallel simulation and distributed simulation. It is written in $\mathrm{C}++$ for performance. Among the cons, OMNET++ has a poor documentation and its models of mobility and physics are limited. On the other hand, NS3 is developed in C++ since 2006 and its models supports both IP and non-IP networks, and a wide range of wireless protocols. Accuracy of the models provided by the tool can also be easily validated and models are welldocumented. We use in our architecture OMNET++ for modelling the simulated OS stack and NS3 for the mobility and the physical models (Figure 2b).

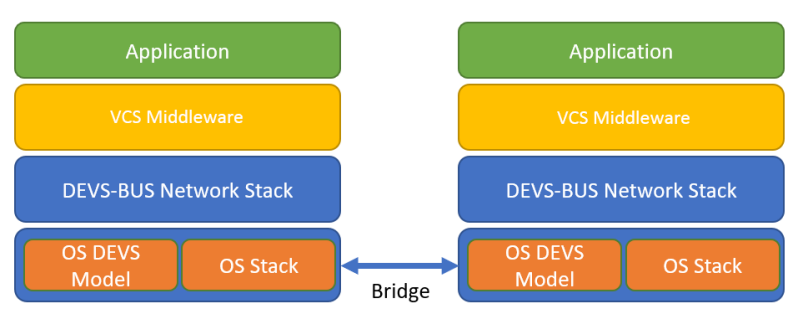

(a) Simulation Environment.

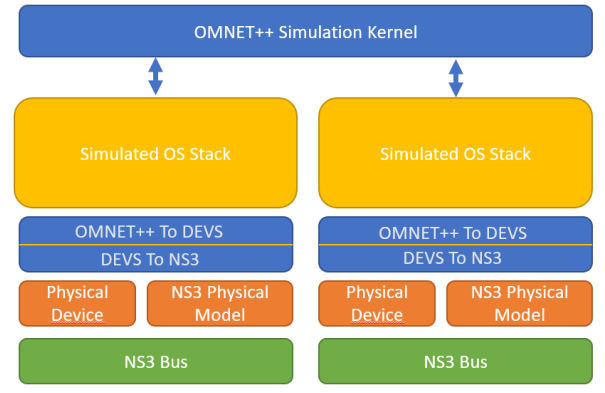

(b) Simulation Core.

Figure 2: Virtual Communication Framework.

The most important part of our VCS Simulation Core concerns the OMNET++ to NS3 Bus. It is composed by two distinct DEVS coupled model: one the one hand, the first bridge translates the output of OMNET++ to DEVS output. Then, the output of DEVS is translated into a message understandable by NS3. This message is then carried to the underlayer which corresponds to the physical model. In the case of full simulation mode, the NS3 physical model is used. The message is then translated into a DEVS message and carried through the NS3 Bus to the VCS Simulation Environment of the other node. If the physical device is implemented, therefore the message use the real implementation of the devices (i.e. the hardware). For this part, the OMNET++ Simulation Kernel is used to synchronize the processing and the simulation of the OMNET++ models (as if the Kernel controls the communication). The protocol converter is defined as followed:

- $\quad X, Y=\{m \in M\}$ where $M$ is all the possible messages in input or output of OMNET++ and NS3 models;

- $\quad S$ contains only transitory states; each state correspond to an event $x$ or $y$;

- $t a(s)=0 \forall s \in S$; 


\subsection{DEVS Integrator for Heterogeneous Environment}

Finally, our DEVS integrator architecture is depicted in Figure 3. Each part is encapsulated in a DEVS coupled model. Each layer can be cross-simulated using the desired level of abstraction along the communication pipeline (using the different simulation models). Moreover, in the opposite of previous work in which simulators interoperate independantly, meaning each DEVS coupled model was simulated by its own simulator, our architecture allows keeping the coupling between devices. The clock between the simulators is managed only by the VCS Simulation Environment which works along to the OMNET++ Simulation Kernel. The Environment keeps also a spatial representation of all the elements and maintains the topology of the simulation. Any External Co-Simulation Environment (like a 3D simulator) communicates with the simulation environment through a specific DEVS protocol which depends on this co-simulation environment. When a device enters in the simulation, a new DEVS model is instantiated in the VCS Simulation Environment. The coupling with the other models is done according to the topology of the network computed by VCS middleware. In this way, when a packet needs to be transmitted from a device to another in the OMNET++ simulation, it is transmitted through the direct coupling to the VCS which handles the routing. The VCS computes the next neighbor and send the message back to the corresponding OMNET++ model. We mean that an instance of a node in OMNET++ is directly coupled to its representant in the simulation environment (a node is a composition of the VCS and a model in the OMNET++ environment). In the same way, the intermediate stack and physical computations can be performed using the VCS Simulation Core which groups the correspond NS3 models, or the Testing Environment which are composed by real implementations software or hardware.

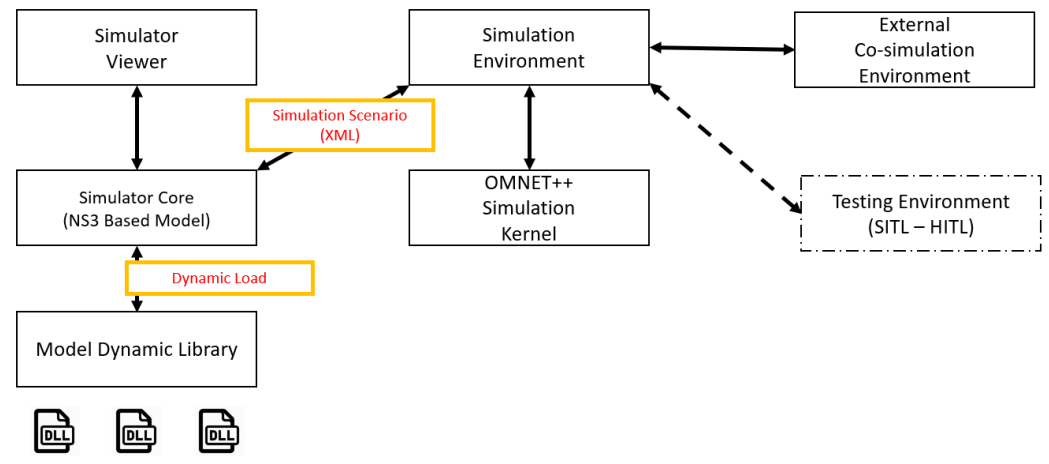

Figure 3: MANET-Based Heterogeneous Simulation Architecture.

\subsection{Integrated Software-in-the-Loop and Hardware-in-the-Loop Paradigms}

Software-in-the-Loop (SITL)/Hardware-in-the-Loop (HITL) Environement allows the use of real hardware when checking the architecture. The testing environment is also controlled by a scheduler which synchronizes date between the simulation environment and the real hardware. An example of Software-in-the-loop or Hardware-in-the-loop testing configuration is shown in Figure 4.

We can distinguish three types of nodes:

- Real nodes are devices which are executing the real communication pipeline through the VCS;

- Virtual nodes are fully simulated devices; a real implementation of the VCS is running to handle communication, but a part or the entire devices is modelled;

- Hybrid nodes are devices whose the model is composed by real implementation and simulated model. For instance, mobility can be fully simulated while the physical layer can be part simulated. Simulation can help to extrapolate values in this case. 


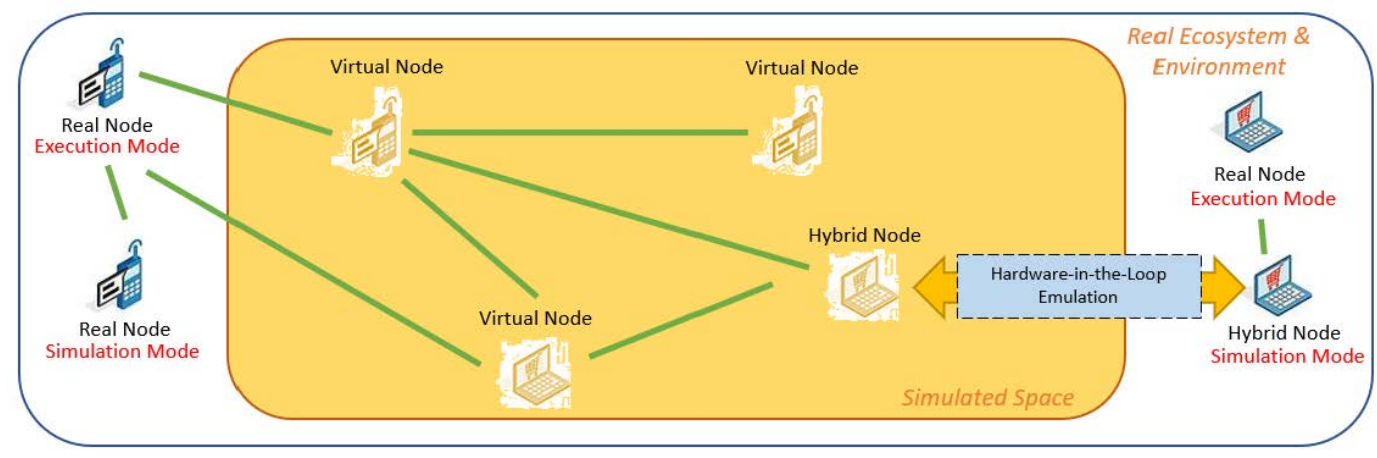

Figure 4: MANET-Based Simulation Infrastructure.

From the point the of view of an end-user application, the communication is entirely transparent. This means that normal application can be used to test all the protocols of the network, and the results can be compared with those obtained in real conditions in order to perform triple validation: validation of the simulation model, validation of the MANET-based infrastructure, and validation of the application.

\section{VALIDITY, RESULTS AND DISCUSSION}

\subsection{Validation Protocol and Results}

In order to validate our approach, and while it is difficult to gather accurate data in the case of emergency, we develop a small protocol which consists on comparing the results obtained using our VCS in normal (execution) mode, the VCS in simulation mode, and a pure NS3 model because of its capability of using the network bridge to transmit data to a simulator through the OS stack. We develop a small chat application which tries to establish communications through the MANET using a standard routing protocol, and send continuously data by varying the distance between nodes, speed and manual interferences (which represents lag because of the connection settings or computation latency). Therefore, in simulation mode, a DEVS Bus translates messages and events incoming and outcoming to any part of the simulator. In normal mode, each DEVS Bus are disabled in order to allow direct communications (from the implemntation point of view, it is only a message forwarding). Simulation parameters are summurized in Table 1. Figure 5a shows the throughput when all the nodes try to communicate between each other randomly. Each value is computed by taking the average of the speed for each transmission. In this case, NS3 uses an ideal model which doesn't show the variation of the speed. The VCS simulation model simplifies also a lot of computation, but follows the tendency. However, we need to take carefully this result while there is no guarantee about the stability of the conditions of the experiment.

Table 1: Simulation Protocol.

\begin{tabular}{rr}
\hline Parameters & Values \\
\hline Scales & $1-20$ nodes \\
Simulation Time & $300 \mathrm{~s}$ \\
Simulation Area & Mobility \\
Transmission Range & No \\
Bandwith Limit & $250 \mathrm{~m}$ \\
\hline
\end{tabular}

Another measure is more related to functional aspect of the simulators. Indeed, the goal of this experiment is to measure the number of generated events by the three models, independantly of their importance. Events 
can be connection, disconnection, lost of connection, send, receive, and neighbor discovering. Again, the NS3 model evolves likely the two other curves but the number of observed events is far from the real one. Finally, in the case of latency, the three curves follows the same distribution, but it is not representative due to the low number of nodes.

The last aspect concerns the functional validation of the simulator. In this case, the goal is to compare the behaviour of the VCS in simulated environment with its behaviour in executed instance (we recall that the VCS is always in execution mode). We generate random changes in the communication topology at random time (for instance, a hard losts of connection which implies a change of the topology). We measure the difference of behaviours of the client application in the two configurations and the time the simulator needs to adapt itself to the new situation. Figure $5 \mathrm{c}$ and $5 \mathrm{~d}$ shows the difference between the time needed to computed a change, and the time computed by the simulation.
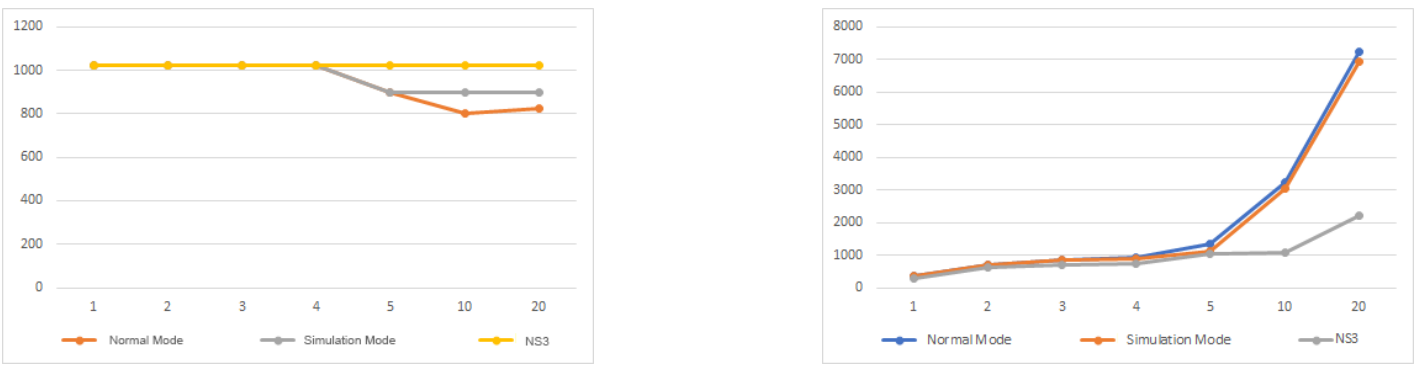

(a) Bandwith variation (in kbps) between NS3, Executed, (b) Number of observable events generated by the VCS and Simulated mode when increasing the number of com- models. Observable events includes connection, disconmunicating nodes. nection, send, receive, and neighbor discovering.
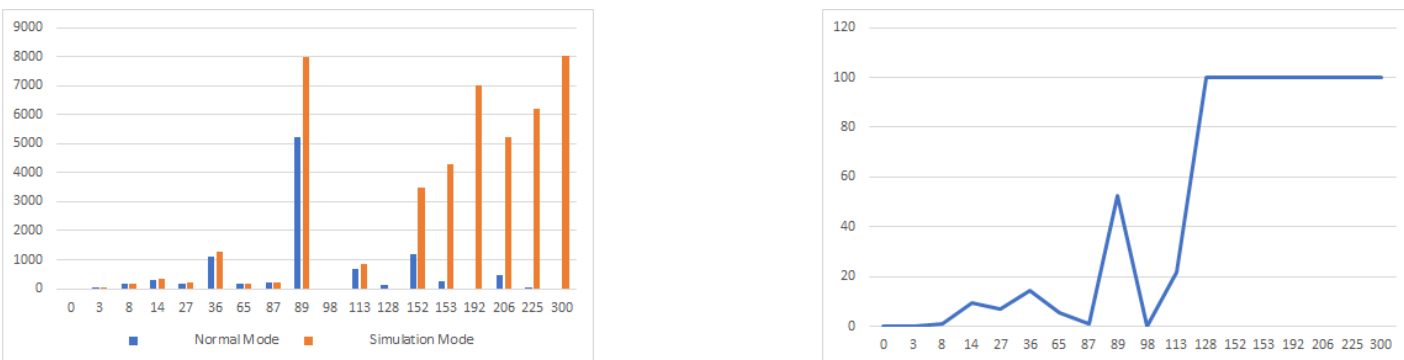

(c) Time needed to compute the change between normal (d) Error rate between the time needed for processing the mode and simulation mode. In simulation mode, the time change and the time computed during the simulation. corresponds to the date of the next event.

Figure 5: Simulation results.

We see that for small variations which doesn't involve a hard recomputation of the topology, the error between the time needed to compute a change and the time computed by the simulator to operate the change is under $10 \%$. This variation is caused by the oversimplified physical model or from the drivers (the only part we don't model in full simulation mode). However, this can be easily measured and used as a control parameter during the simulation. However, as expected, when the time needed to compute a change in simulation exceeds the time of the real communication computation, the simulator enters in an inconsistant state. Indeed, at $t=89$, the change needs more than 8 seconds to be computed by the simulator, while the next event was already arrived in the system. Therefore, a gap between the behaviour of the real implementation and the simulation was created. This gap cannot be corrected, that's why the simulator provides wrong results after this event. Following this result, we can easily see that our simulation is almost able to reproduce 
the behaviour of an application in operational conditions. This means that it can be used to check the consistency of the MANET protocol, but also to validate software using simulation and execution.

Finally, we can summuarize in Table 2 the differences between our approach and a simulator like NS3. Nevertheless, both architecture are designed to work in complementary manner while our proposed architecture integrates existing MANET simulators.

Table 2: Comparison between VCS Simulator and NS3.

\begin{tabular}{lll}
\hline & VCS Simulator & MANET Simulator (NS3 Only) \\
\hline Language & C++ & C++ \\
\hline Paradigm & DEVS & Discrete-Event \\
\hline Model & 7 layers & Network, Data Link, Transport \\
\hline Hardware-in-the-Loop & Yes & Through the TAP adapter only \\
\hline Emulation mode & Yes & No \\
\hline Co-Simulation & Yes & No \\
\hline Parallelism and Distributed & No (future works) & Yes \\
\hline Formal V\&V support & In specific cases & No \\
\hline
\end{tabular}

\subsection{Discussion about the Topology Management and Space Partitioning}

Theoritically, our architecture allows us to make the topology of the communication simulation match the topology of the simulation, making orders of events more natural (when an event is triggered, the consequences on the upper/lower layer are directly computed). However, this advantage becomes a major drawback in the case of high mobility.

In our exemple, the environment is handled by a unique atomic model coupled to all the other nodes. This atomic model handles the propogation of physical waves, the position of nodes and the simulation of physical phenomena. Nodes are directly intercoupled between them, as a result of the neighbor discovering process of the VCS. This coupling is computed at initialization. Connection changes then have a heavy cost, while they imply to restart all the simulation without losing the ordered queued events. Indeed, DEVS theory doesn't allow change of the topology during the simulation, because all the queued future non-processed events can change when a new coupling is performed. A workaround consists on a coupling using space partitioning (Figure 6). When the simulation is initialized, the space is divided into areas using a partitioning algorithm like the Binary Space Partitioning Tree (BSP-Tree) or the Quadtree (Aluru 2004). Explanation about the partitioning is out of the scope of this paper. Each device is then associated to a DEVS coupled model which represents a region. When the simulation is performed for moblity devices, the device simulator sends its event to the region simulator which forwards the communications to the right receiver. For instance, considering the example in Figure 6, if Device 1 wants to communicate with Device 3, admitting that there is a route to the Device 2, the message is sent to Sub-Region 1 which forwards the message to the Device 2 (natural topology network coupling), which sends it back to the Sub-Region 1, which will dispatches it to the Sub-Region 2 (spatial coupling), and finally to the Device 3.

In this case, the time of simulation is not affected but a performance overhead appears. This overhead slows down the simulation, but doesn't imply any errors if the time of computation of the simulation remains under the time of computation of the communication algorithm. In other words, if the communication needs $10 \mathrm{~ms}$ to be performed and the simulation needs more than $10 \mathrm{~ms}$ to compute the transmission, an 


\section{Yacoub}
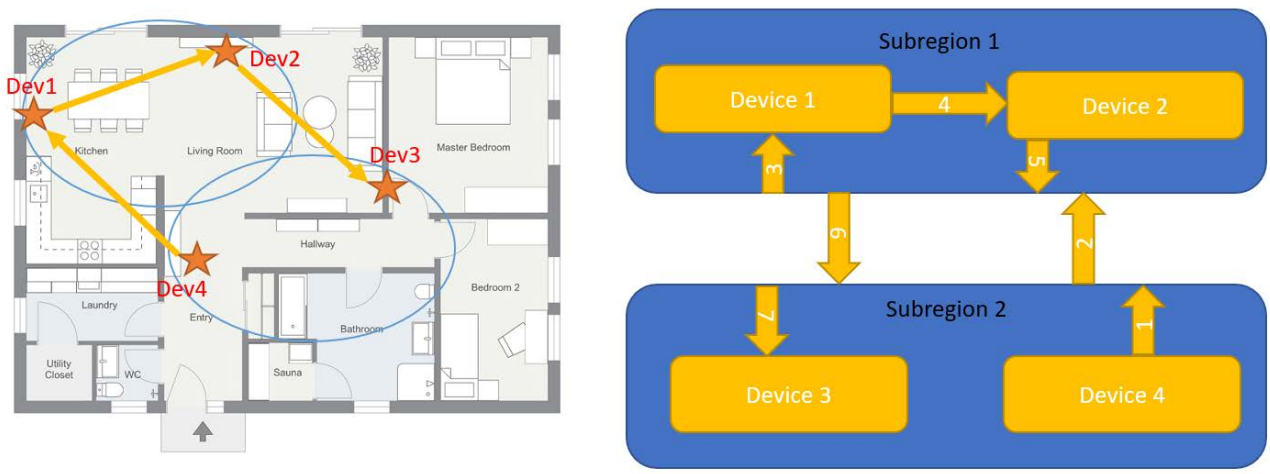

Figure 6: Space Partitionning. On the left, devices are grouped in space partition using a BSP Tree Algorithm. On the right, the simulation topology shows how the message is processed when the Device 4 sends a message to the Device 3 (in the case where there is no direct link betweent the Device 4 and 3 in the MANET).

erroneous outcome occurs. In large scalable network and complex environment, this may eventually be a major drawback. The use of efficient partitioning algorithms or distributed simulations should be considered to overcome this problem.

\section{CONCLUSION}

This paper tries to illustrate the benefits of our proposed architecture for integrated simulation of MANETbased infrastructure. Our implementation takes advantages of the power of the DEVS theory, coupled to network simulators: NS3 and OMNET++. The VCS allows modelling and checking of the routing protocols, while NS3 and OMNET++ allows simulation of different physical parts. Simulation scenario directly integrate final end-user software. At any step of the development, modellers can adapt:

- the granularity of the simulation (the right level of details), by deciding to execute, simulate or emulate one or several parts of the network;

- the accuracy of the simulation implied by the level of abstraction of the physical model;

- the V\&V of the simulation model: end-user software, simulator and protocols can be checked using our approach.

Models and real systems can be swapped in a trasparent co-simulation environment and adapted at each step of the development of the simulator. Real hardware can be integrated using a HIL paradigm, and cosimulation environment allows use of 3D visualization, virtual reality, or augmented reality simulation for instance. The only limit of this architecture is the capability to build a DEVS-Bus for the target co-simulator.

The major drawback concerns the scalability. Due to the restrictions induced by the cosimulation environment, some scenarios cannot be simulated when the move of nodes become too complex. Indeed, when the time to compute the simulation becomes greater than the time to carry data from one node to another, the simulation becomes unefficient. Indeed, while positions of nodes are replicated both in network simulator and environment simulator, desynchronizations may happen in these cases, leading to inconsistant situations. Future works will address the improvement of the computation of mobile nodes, which can be achieved by integrating other DEVS-based formalisms like Cellular DEVS (Cell-DEVS) or Dynamic Structure DEVS (DS-DEVS). Full integration of combined formalisms is an interesting point to develop, since they will allow combined checking and improve the reliability of verification and validation processes. 
Yacoub

\section{ACKNOWLEDGMENTS}

I thank Alexy Torres and Julien Carayol for their feedbacks, Pr. Gabriela Nicolescu and Pr. Claudia Frydman for their support. This project was funded by MITACS and done in collaboration with Humanitas Solutions.

\section{REFERENCES}

Aluru, S. 2004. Quadtrees and Octrees. Chapman and Hall/CRC.

Andel, T. R., and A. Yasinsac. 2006, July. "On the credibility of manet simulations". Computer vol. 39 (7), pp. $48-54$.

Giambiasi, N. 2009. "From Sequential Machines to DEVS Formalism". In Proceedings of the 2009 Summer Computer Simulation Conference, pp. 216-222, SCS.

Hogie, L., P. Bouvry, and F. Guinand. 2006. “An Overview of MANETs Simulation”. Electron. Notes Theor. Comput. Sci. vol. 150 (1), pp. 81-101.

Kiess, W., and M. Mauve. 2007. "A survey on real-world implementations of mobile ad-hoc networks". Ad Hoc Networks vol. 5 (3), pp. 324 - 339.

Kim, Y. J., and T. G. Kim. 1998. "A heterogeneous simulation framework based on the DEVS BUS and the high level architecture". In 1998 Winter Simulation Conference. Proceedings, pp. 421-428.

Kurkowski, S., T. Camp, and M. Colagrosso. 2005, October. "MANET Simulation Studies: The Incredibles”. SIGMOBILE Mob. Comput. Commun. Rev. vol. 9 (4), pp. 50-61.

Mallapur, S. V., and S. R. Patil. 2012. "Survey on simulation tools for mobile ad-hoc networks". International Journal of Computer Networks and Wireless Communications (IJCNWC) vol. 2 (2).

Manpreet, and J. Malhotra. 2014. "A survey on MANET simulation tools". In 2014 Innovative Applications of Computational Intelligence on Power, Energy and Controls with their impact on Humanity, pp. 495498.

Mohammed, A., and A. Al-Ghrairi. 2019. "Differences between Ad Hoc Networks and Mobile Ad Hoc Networks: A Survey". Journal of Southwest Jiaotong University vol. 54, pp. 12.

Reina, D. G., M. Askalani, S. L. Toral, F. Barrero, E. Asimakopoulou, and N. Bessis. 2015. "A Survey on Multihop Ad Hoc Networks for Disaster Response Scenarios". International Journal of Distributed Sensor Networks vol. 11, pp. 647037.

Riley, G. F., and T. R. Henderson. 2010. The ns-3 Network Simulator, pp. 15-34. Berlin, Heidelberg, Springer Berlin Heidelberg.

Sargent, R. G. 2001. "Some approaches and paradigms for verifying and validating simulation models". In Simulation Conference, 2001. Proceedings of the Winter, Volume 1, pp. 106-114.

Varga, A., and R. Hornig. 2008. "An Overview of the OMNeT++ Simulation Environment". In Proceedings of the 1st International Conference on Simulation Tools and Techniques for Communications, Networks and Systems \& Workshops, pp. 60:1-60:10, ICST.

Yacoub, A. 2019. "Virtual Communication Stack: Towards Building Integrated Simulator of MANET-based Infrastructure for Disaster Response Scenarios". Preprint.

Zeigler, B. P., A. Muzy, and E. Kofman. 2019. Theory of Modeling and Simulation. 3rd ed. Academic Press.

\section{AUTHOR BIOGRAPHIES}

AZNAM YACOUB holds a PhD in Computer Science from Aix-Marseille University. He's a postdoctoral fellow at Polytechnique Montreal. His research interests are mainly Modelling and Simulation Theory, and Verification and Validation of Software and Systems. His email address is aznam.yacoub@polymtl.ca. 